\title{
Treatment of Anterior Cruciate Ligament Tibial Avulsion Fracture in Children with Suture Anchor Fixation Outside The Tunnel: A Retrospective study
}

Sunyu Chen ( $\nabla$ hunson2009@163.com )

Fuzhou Second Hospital

\section{Zhanhao Xiao}

Fuzhou Second Hospital Affiliated to Xiamen University

Jiankun Wang

Fuzhou Second Hospital Affiliated to Xiamen University

Research article

Keywords: children, anterior cruciate ligament, tibial fracture, arthroscopy

Posted Date: October 7th, 2020

DOI: https://doi.org/10.21203/rs.3.rs-84453/v1

License: (c) (i) This work is licensed under a Creative Commons Attribution 4.0 International License.

Read Full License 


\section{Abstract}

Background: Anterior cruciate ligament $(\mathrm{ACL})$ tibial avulsion fractures are a special $A C L$ injury type, classified as intra-articular fractures. Presently, the main treatment method is arthroscopic surgery, and various fixation methods are available, including use of steel wires, high-strength sutures, cannulated screws, anchors, Kirschner wires, etc. Joint fixation using wire binding could cut through the bone tunnel. We aimed to evaluate the clinical efficacy of a novel method involving suture anchor fixation outside the tunnel to treat anterior cruciate ligament (ACL) tibial avulsion fractures in children.

Methods: This retrospective study analyzed the data of 42 pediatric patients ( 26 boys and 16 girls; age: 7-13 years) with ACL tibial avulsion fractures. Based on the Meyers-McKeever-Zaricznyj classification of fractures, 22, 14, and 6 patients had types II, III, and IV fractures, respectively. All patients underwent arthroscopic surgery for ACL tibial avulsion fracture; during surgery, double tibial tunnels were established, and high-strength sutures were passed through the tunnels and fixed externally with an anchor. The clinical outcome was evaluated by assessing the pre- and post-operative knee joint range of motion (ROM) and by using the Lysholm knee function score and International Knee Documentation Committee (IKDC) score. Post-operative computed tomography and magnetic resonance images were reviewed to determine the status of fracture displacement, healing, and epiphyseal damage.

Results: All 42 patients were followed up for 20-36 months (average of 27.8 months). Knee ROM increased from $48.2^{\circ} \pm 21.7^{\circ}$ pre-operatively to $131.6^{\circ} \pm 8.7^{\circ}$ at the final follow-up ( $t=23.119, P=0.000$ ). The Lysholm knee function score increased from $37.6 \pm 5.2$ points pre-operatively to $90.1 \pm 6.3$ points postoperatively, representing a significant improvement $(t=41.651, P=0.000)$. The IKDC score improved from $43.3 \pm 7.5$ points pre-operatively to $91.3 \pm 5.7$ points post-operatively ( $t=45.521, P=0.000)$. The imaging findings indicated that the fractures healed with displacement and there was no significant epiphyseal damage.

Conclusions: Suture anchor fixation outside the tunnel to treat ACL tibial avulsion fracture in children reduces the cutting action of the sutures on the tunnel, minimizes epiphyseal damage, involves a simple procedure, offers firm fixation, and effectively improves knee function. This approach can enable early functional rehabilitation and achieve satisfactory clinical efficacy.

\section{Background}

An anterior cruciate ligament (ACL) tibial avulsion fracture is a special type of ACL injury that mostly occurs in children aged 8-14 years and accounts for $14 \%$ of $A C L$ injuries. ${ }^{1}$ Owing to the traction of the $\mathrm{ACL}$, the fracture is easily displaced and can become trapped in the intercondylar notch, which can affect the flexion and extension of the affected limb. According to the Meyers-McKeever-Zaricznyj classification, surgical reduction is necessary for type II-IV fractures.

In adult patients, ACL tibial fractures are currently treated using cannulated screws, suture anchors, steel wires, high-strength sutures, and absorbable screws, with a satisfactory therapeutic efficacy in clinical 
reports. ${ }^{2-5}$ However, as children are still growing and developing, and their treatment principles differ from those of adults, special techniques in fracture reduction and fixation, with the aim of avoiding iatrogenic injuries to the epiphysis during surgery when possible, need to be developed. The suture binding technology is widely used for ACL tibial avulsion fracture at present. ${ }^{6}$ Previous studies showed that it is safe and feasible to reconstruct the ACL through the epiphyseal tunnel without varus or valgus deformity and leg shortening. ${ }^{7-8}$ The use of suture anchors outside the tunnel is a novel method, and it can prevent high-strength sutures from cutting through the bone tunnel. This study aimed to evaluate the clinical efficacy of using suture anchor fixation outside the tunnel in treating ACL tibial avulsion fractures in children. Our hypothesis was that the application of suture anchors outside the tunnel to fix ACL tibial avulsion fractures would have advantages and would result in good fracture reduction.

\section{Methods}

\section{General information}

This retrospective study analyzed the data of 42 children with ACL tibial avulsion fractures who were admitted to our hospital between March 2014 and November 2017. This study was approved by the ethics committee of Fuzhou Second Hospital (No: FZSE2014039). Written informed consent was provided by the patients and their parent or legal guardian prior to participating in the study.

\section{Inclusion criteria}

The inclusion criteria were as follows: (1) age between 7 and 13 years; (2) ACL tibial avulsion fractures of Meyers-McKeever-Zaricznyj types II-IV ( $\leq 3$ weeks post-injury); and (3) treatment with the same surgical method (extra-tunnel suture anchor fixation).

\section{Exclusion criteria}

The exclusion criteria were as follows: (1) old fractures ( $\geq 3$ weeks) with a healing deformity that ends with an unreducible fracture; (2) other ligament injuries or type III meniscus injuries; and (3) cartilage injuries in other parts of the knee joint.

\section{Surgical technique}

After successful induction of anesthesia, a tourniquet was applied at the proximal thigh of the affected limb. The patient was then placed in a supine position, routinely disinfected, and draped, and the knee arthroscopy anteromedial and anterolateral approaches were established. Second, the synovial membrane, adipose tissue, and hematoma surrounding the fracture end were debrided. The meniscus and transverse ligament were retracted using a probe hook to fully expose the ACL tibial avulsion fracture (Fig. 1A).Third, using the Lasso technique, two high-strength sutures of different colors were passed posteriorly around the ACL; then, both ends of the sutures were pulled anteriorly. Fourth, the anterior and anteromedial edges of the tibial avulsion fracture were fixed using ACL fixators (Fig. 1B), drilled using 2.0$\mathrm{mm}$ Kirschner wires, and pierced with a lumbar puncture needle. Then, a thin wire (Lasso suture) was 
guided through the tunnel with both ends of the same high-strength suture passing though the same tunnel to apply downward pressure on the upturned bone (Fig. 1C). Fifth, an absorbable anchor (DepuySynthes, Raynham, MA, US) was implanted at the tunnel exit, and the fracture was reduced by a downward traction of the pull sutures (Fig. 1D). The pulley technique was used to knot the pull sutures and anchor sutures of the same color, and the two pull sutures were fixed on the anchor (Fig. 1E). Finally, the fracture end was stable after mobilizing the knee joint, and the wound was closed layer by layer.

\section{Post-operative care}

To protect the knee, an extension splint was used for 4 weeks with partial weight-bearing and crutches were used after the surgery. Quadriceps strength training and passive joint flexion exercises began on the first day after the surgery. The passive range of motion (ROM) of the knee joint was maintained within $0^{\circ}-90^{\circ}$. Full ROM and full weight-bearing exercises were allowed on the fourth week after surgery.

\section{Outcome evaluation indicators}

Post-operative computed tomography and magnetic resonance images were reviewed to determine the status of fracture displacement, healing, and epiphyseal damage. Preoperative and final follow-up joint ROM, the Lysholm score, and the International Knee Documentation Committee (IKDC) score were assessed to evaluate the clinical outcomes.

\section{Statistical methods}

The SPSS19.0 (SPSS Inc., Chicago, IL, US) statistical software package was used to perform statistical analyses on the data. Measurement data that were normally distributed or approximately normally distributed were expressed as mean \pm standard deviation. Comparisons of pre- and post-operative observational indicators were performed using paired samples t-test. The significance level of testing was set at $a=0.05$.

\section{Results}

\section{General information}

There were 26 boys and 16 girls, with a mean age of 12 years (range, 7-13 years). Based on the MeyersMcKeever-Zaricznyj classification of fractures, 22, 14, and 6 patients had type II, III, and IV fractures, respectively.

\section{Basic operative conditions}

All patients in this study successfully completed the surgery, and the operative time ranged from $0.5 \mathrm{~h}$ to $1 \mathrm{~h}$. There were no abnormalities or complications postoperatively in all cases, and the length of hospital stay was 3-5 days.

\section{Efficacy evaluation}


All 42 patients were followed up for 25-36 months. The imaging findings at the final follow-up showed the absence of fracture displacements, deformities in healing, or damages to the epiphysis (Fig. 2).

Knee joint ROM increased from $48.2^{\circ} \pm 21.7^{\circ}$ pre-operatively to $131.6^{\circ} \pm 8.7^{\circ}$ at the final follow-up $(t=$ $23.119, P=0.000)$. The Lysholm knee function score significantly improved from $37.6 \pm 5.2$ points preoperatively to $90.1 \pm 6.3$ points post-operatively $(t=41.651, P=0.000)$. The IKDC score significantly improved from $43.3 \pm 7.5$ points preoperatively to $91.3 \pm 5.7$ points $(t=45.521, P=0.000)$. All measured indicators showed statistically significant differences when the values obtained pre- and post-operatively were compared (Table 1).

Table 1

The pre-operative and final follow-up knee joint status of the 42 pediatric cases with ACL tibial avulsion fracture (mean \pm standard deviation)

\begin{tabular}{|lllll|}
\hline Time(min) & Cases & Knee joint ROM $\left(^{\circ}\right)$ & Lysholm score(points) & IKDC \\
\hline Pre-operative & 42 & $48.2 \pm 21.7^{\circ}$ & $37.6 \pm 5.2$ & $43.3 \pm 7.5$ \\
\hline Final follow-up & 42 & $131.6 \pm 8.7^{\circ}$ & $90.1 \pm 6.3$ & $91.3 \pm 5.7$ \\
\hline$t$ & 23.119 & 41.651 & 45.521 \\
\hline$p$ & 0.000 & 0.000 & 0.000 \\
\hline \multicolumn{2}{l}{$\begin{array}{l}\text { Abbreviations: ACL, anterior cruciate ligament; ROM, range of motion; IKDC, International Knee } \\
\text { Documentation Committee }\end{array}$} \\
\hline
\end{tabular}

\section{Discussion}

In this study, arthroscopy was used to perform suture anchor fixation outside the tunnel to treat children with ACL tibial fractures. The follow-up period was 25-36 months. No deformities or fracture nonunion were found in the affected limb; thus, the clinical outcome was satisfactory. The key points of this technique are as follows: (1) synovial tissue and hematoma surrounding the fracture were debrided, and the bone bed was freshened to avoid affecting fracture reduction; (2)fixation was performed using ACL tibial fixators, with the anterior and medial edges of the fracture as the chosen fixation sites. Fine Kirschner wires were used to establish the tunnels, and sutures were guided through the tunnels. Following the direction of the $\mathrm{ACL}$, traction was first applied to the medial pull suture to correct the lateral displacement, then to the anterior pull suture to apply downward pressure on the fractured bone;(3) during reduction, the anterior meniscus and transverse ligament are retracted using probe hooks to avoid affecting fracture reduction; and (4) under arthroscopic monitoring, the double pulley technique was used to adjust the tension of the high-strength suture.

An ACL tibial avulsion fracture is a type of ACL injury. The flexion and rotational force of the knee joint can result in an ACL tibial avulsion fracture and displacement, ligament contracture, limited flexion and extension of the knee joint, and impaired movement. Currently, such displaced fractures are often treated using arthroscopic surgery, and a wide variety of fixation methods are available, including steel wires, 
high-strength sutures, cannulated screws, anchors, Kirschner wires, and so on. Steel wires offer poor toughness and non-elastic fixation; hence, breakage can occur easily during knee flexion and extension. ${ }^{9,10}$ Kirschner wire fixation causes minimal damage, but it does not offer sufficient holding power, which can easily lead to the loosening or detachment of internal fixation, thereby resulting in fracture re-displacement and affecting the function of the affected limb. ${ }^{11}$ Currently, the most commonly used method in clinical settings is the technique of using cannulated screws, which generally involves utilizing two screws to fix the fractured bone. This method is especially effective in the fixation of larger bone fractures. ${ }^{12}$ However, for pediatric patients, the relatively large diameter of cannulated screws means that the cutting action of the thread when inserting the screw can cause a more significant damage to the epiphysis. Moreover, ACL tibial fractures are smaller in children than in adult patients, which implies that screwing in and removing the fixators will cause a greater damage to the ACL tibial spine. Therefore, this method should be used with caution in pediatric patients. ${ }^{13}$ Fracture fixation with high-strength sutures are also widely used in clinical practice. It is advantageous for the fixation of comminuted ACL tibial fractures and does not require a second surgery to retrieve the fixator. However, slippage of the sutures may occur to different degrees during knee flexion and extension, which may cause cutting to the tunnel, thereby preventing early functional recovery. ${ }^{14,15}$ Internal fixation with suture anchors is one of the surgical approaches for treating ACL tibial avulsion fracture. In et al. used intraarticular suture anchors to fix the fractured bone and obtained a satisfactory clinical efficacy. ${ }^{16}$ However, they encountered difficulty in adjusting the tension of the intra-articular suture, and excessively large knots may irritate the synovium or affect joint extension. Yao et al. used intra-articular fixation with double row anchors to treat ACL tibial fractures and achieved satisfactory clinical efficacy. ${ }^{17}$ However, for pediatric patients, the relatively large diameter of the lateral row anchors implies that pre-drilling and screwing in the anchor may lead to epiphyseal damage.

Unlike in adults, the proximal tibial epiphyseal plate in children plays a crucial role in the growth and development of the lower limbs. This is of particular importance when selecting the surgical method and fixation for intra-articular fractures in this group. Jang et al. found that the 8-mm bone tunnel only damages $2.5 \%$ of the growth area of the tibial epiphyseal plate, and the damage area will decrease with age. ${ }^{18}$ Thus, avoiding epiphyseal damage as much as possible is our top priority. Not only should we consider the healing of the fracture during the post-operative follow-up, but we should also pay attention to whether there is an epiphyseal damage and an abnormal development in the affected limb. The followup period should, therefore, be extended accordingly. Sinha et al. adopted an epiphyseal plate evasion method for the suture fixation of ACL tibial avulsion fractures in children, and the early clinical outcomes were satisfactory. ${ }^{19}$ Using arthroscopic techniques, Liu et al. drilled across the epiphyseal plate and performed figure-8 suture fixation to treat ACL tibial avulsion fractures in children. ${ }^{20}$ No deformities were found in the lower limbs of the patients during the 2-year follow-up.

The surgical method adopted in the present study has the following advantages: (1) drilling with smooth, fine Kirschner wires can avoid epiphyseal damage caused by the drilling of coarse screw threads; (2) the same suture remains in the same tunnel, which prevents cutting of the tunnel during knee flexion and 
extension; (3) the surgical procedures are simple and do not require complicated winding of sutures. The two high-strength sutures are passed around posteriorly to the ACL tibial spine and pulled anteriorly. Then, the same high-strength suture is passed through the same tunnel to apply downward pressure on the upturned bone, which was subsequently followed by reduction and fixation; (4) suture anchor fixation outside the tunnel avoids the epiphysis, which only causes minimal damage to the epiphysis, and allows the tightening of the high-strength sutures for the effective fixation of the fractured bone; and (5) it involves an extra-articular knot that will not irritate the synovium or affect the flexion and extension of the joint.

\section{Limitations}

Given that the sample size of this study is relatively small, further large-scale follow-up studies are still needed. Furthermore, our technique has only been used in a clinical setting. To confirm the superiority of the extra-tunnel suture anchor fixation method, further biomechanical studies are required.

\section{Conclusions}

The use of arthroscopic reduction monitoring and suture anchor outside the tunnel to treat children with ACL tibial fractures involves simple procedures, results in reliable reduction and fixation, causes minimal epiphyseal damage, and reduces the cutting effect of the high-strength sutures on tissues. Therefore, this approach can enable early functional rehabilitation and achieve satisfactory clinical efficacy.

\section{List Of Abbreviations}

$\mathrm{ACL}$, anterior cruciate ligament; ROM, range of motion; IKDC, International Knee Documentation Committee

\section{Declarations}

Ethics approval and consent to participate: This study was approved by theEthics Committee of Fuzhou Second Hospital(No: FZSE2014039). Written informed consent was provided by the patients prior to participating in the study.

Consent for publication: The manuscript does not contain any individual's data in any form (including personal or identifying details, images, or videos), and consent for publication has been obtained from the parent or legal guardian of the children. Availability of data and materials: All data analyzed in this study has been provided in the manuscript.

Competing interests: The authors declare that they have no competing interests.

Funding: None 
Authors' contributions: SC participated in the design of this study and analyzed and interpreted the patient data. ZX provided the clinical cases. JW provided clinical help and performed the literature search. All authors read and approved the final manuscript.

Acknowledgements: Not applicable

\section{References}

1. Gamboa JT, Durrant BA, Pathare NP, Shin EC, Chen JL. Arthroscopic reduction of tibial spine avulsion: suture lever reduction technique. Arthrosc Tech. 2017;6:e121-6.

2. Shin $\mathrm{CH}$, Lee DJ, Choi IH, Cho TJ, Yoo WJ. Clinical and radiological outcomes of arthroscopically assisted cannulated screw fixation for tibial eminence fracture in children and adolescents. BMC Musculoskelet Disord. 2018;19:41.

3. Scrimshire AB, Gawad M, Davies R, George H. Management and outcomes of isolated paediatric tibial spine fractures. Injury. 2018;49:437-42.

4. Wiegand N, Naumov I,Vámhidy, L, NötLG. Arthroscopic treatment of tibial spine fracture in children with a cannulated Herbert screw. Knee. 2014;21:481-5.

5. Mahar AT, Duncan D, Oka R, Lowry A, Gillingham B, Chambers H. Biomechanical comparison of four different fixation techniques for pediatric tibial eminence avulsion fractures. J Pediatr Orthop. 2008;28:159-62.

6. El Said ANS, Zein AMN, ElShafie M, ElSaid NS, Mahmoud AZ. Arthroscopic single-tunnel pullout suture fixation for tibial eminence avulsion fracture. Arthrosc Tech. 2018;7:e443-52.

7. Swart E, Redler L, Fabricant PD, Mandelbaum BR, Ahmad CS, Wang YC. Prevention and screening programs for anterior cruciate ligament injuries in young athletes: a cost-effectiveness analysis. $\mathrm{J}$ Bone Joint Surg Am. 2014;96:705-11.

8. Kohl S, Stutz C, Decker S, Ziebarth K, Slongo T, Ahmad SS. Mid-term results of transphyseal anterior cruciate ligament reconstruction in children and adolescents. Knee. 2014;21:80-5.

9. In Y, Kwak DS, Moon CW, Han SH, Choi NY. Biomechanical comparison of three techniques for fixation of tibial avulsion fractures of the anterior cruciate ligament. Knee Surg Sports Traumatol Arthrosc. 2012;20:1470-8.

10. Gan Y, Xu D, Ding J, Xu Y. Tension band wire fixation for anterior cruciate ligament avulsion fracture: biomechanical comparison of four fixation techniques. Knee Surg Sports Traumatol Arthrosc. 2012;20:909-15.

11. Zhao QC, Deng XW, Zhou P, Shang XF, Zhu YL. Arthroscopic treatment of tibial intercondylar eminence fractures in children with single Kirschner wire and suture avoiding epiphyseal line fixation technique. Zhongguo Gu Shang. 2015;28:504-7.

12. Leie M, Heath E, Shumborksi E, Salmon L, Roe J, Pinczewski L. Midterm outcomes of arthroscopic reduction and internal fixation of anterior cruciate ligament tibial eminence avulsion. Arthroscopy. 2019;35:1533-46. 
13. Fabricant PD, Osbahr DC, Green DW. Management of a rare complication after screw fixation of a pediatric tibial spine avulsion fracture: a case report with follow-up to skeletal maturity. J Orthop Trauma. 2011;25:e115-9.

14. Strauss EJ, Kaplan DJ, Weinberg ME, Egol J, Jazrawi LM. Arthroscopic management of tibial spine avulsion fractures: principles and techniques. J Am Acad Orthop Surg. 2018;26:360-7.

15. Callanan M, Allen J, Flutie B, Tepolt F, Miller PE, Kramer D, et al. Suture versus screw fixation of tibial spine fractures in children and adolescents: comparative study. Orthop J Sports Med. 2019;7:2325967119881961.

16. In Y, Kim JM, Woo YK, Choi NY, Moon CW, Kim MW. Arthroscopic fixation of anterior cruciate ligament tibial avulsion fractures using bioabsorbable suture anchors. Knee Surg Sports Traumatol Arthrosc. 2008;16:286-9.

17. Yao J, Wang H, Quan S, Feng W, Cai L, Yang M. Suture-bridge fixation under arthroscopy in treatment of tibial eminence avulsion fracture of anterior cruciate ligament in adolescents. Zhongguo Xiu Fu Chong Jian Wai Ke Za Zhi. 2018;32:1402-5.

18. Jang KM, Bae JH, Kim JG, Wang JH. Novel arthroscopic fixation method for anterior cruciate ligament tibial avulsion fracture with accompanying detachment of the anterior horn of the lateral meniscus: three-point suture fixation. Injury. 2013;44:1028-32.

19. Sinha S, Meena D, Naik AK, Selvamari M, Arya RK. Arthroscopic fixation of tibial spine avulsion in skeletally immature: the technique. J Orthop Case Rep. 2017;6:80-4.

20. Liu Y, Sun X, Zhang K, Li G, Ni J. Arthroscopic treatment of anterior cruciate ligament tibial eminence avulsion fracture in adolescents with epiphyseal unclosure. Zhongguo Xiu Fu Chong Jian Wai Ke Za Zhi. 2015;29:725-9.

\section{Figures}



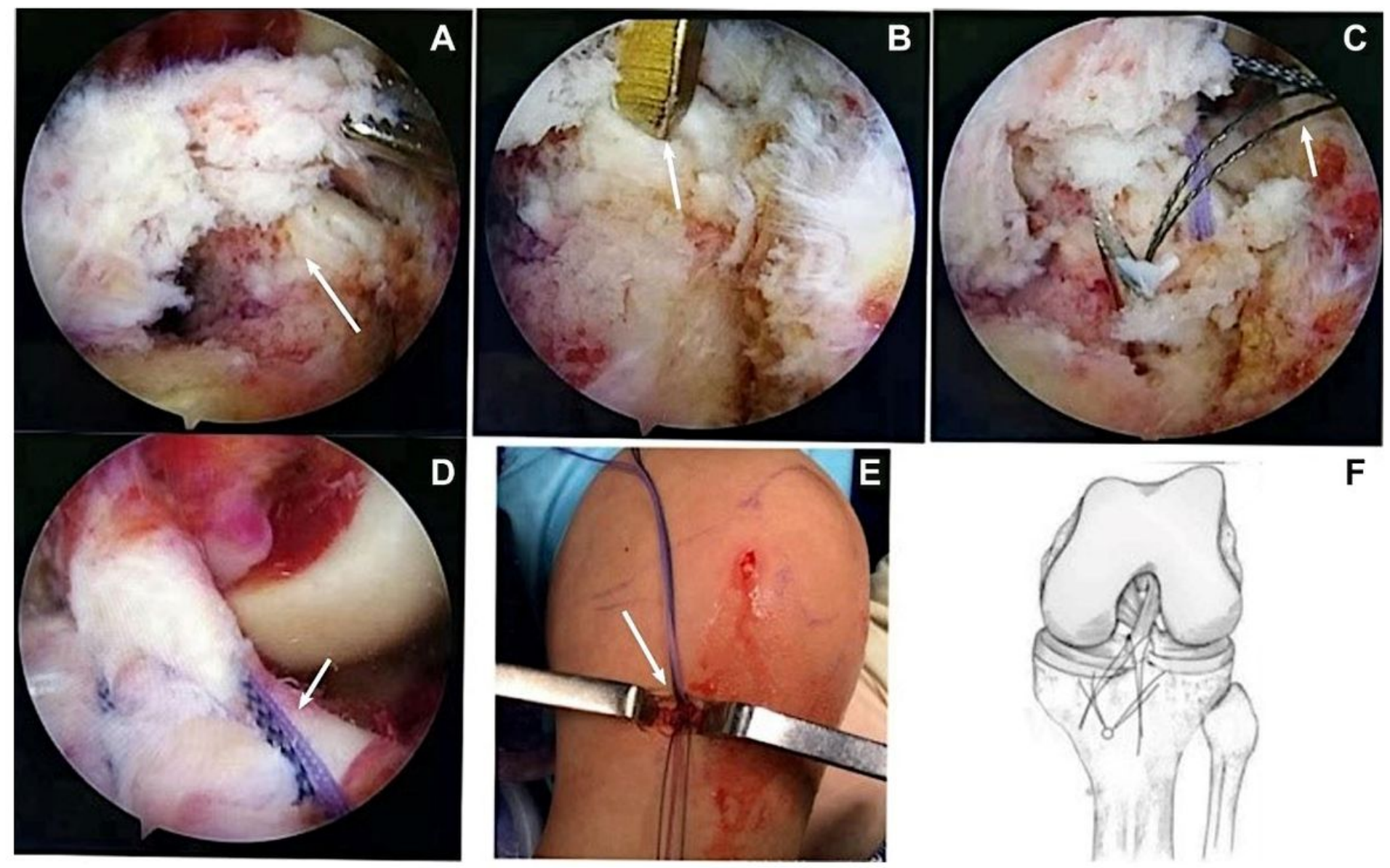

\section{Figure 1}

Suture anchor fixation outside the tunnel to treat anterior cruciate ligament (ACL) tibial avulsion fracture in children. A: ACL tibial fracture displacement under arthroscopy (white arrow). B: ACL tibial fixators fixed on the fracture edge. C: Passage of high-strength sutures with the same color through the same tibial tunnel (white arrow). D: Bone reduced by traction with high-strength sutures (white arrow). E: Fixation of high-strength sutures from the tunnel on the anchors (white arrow). F: Schematic drawings of the surgical procedure. 

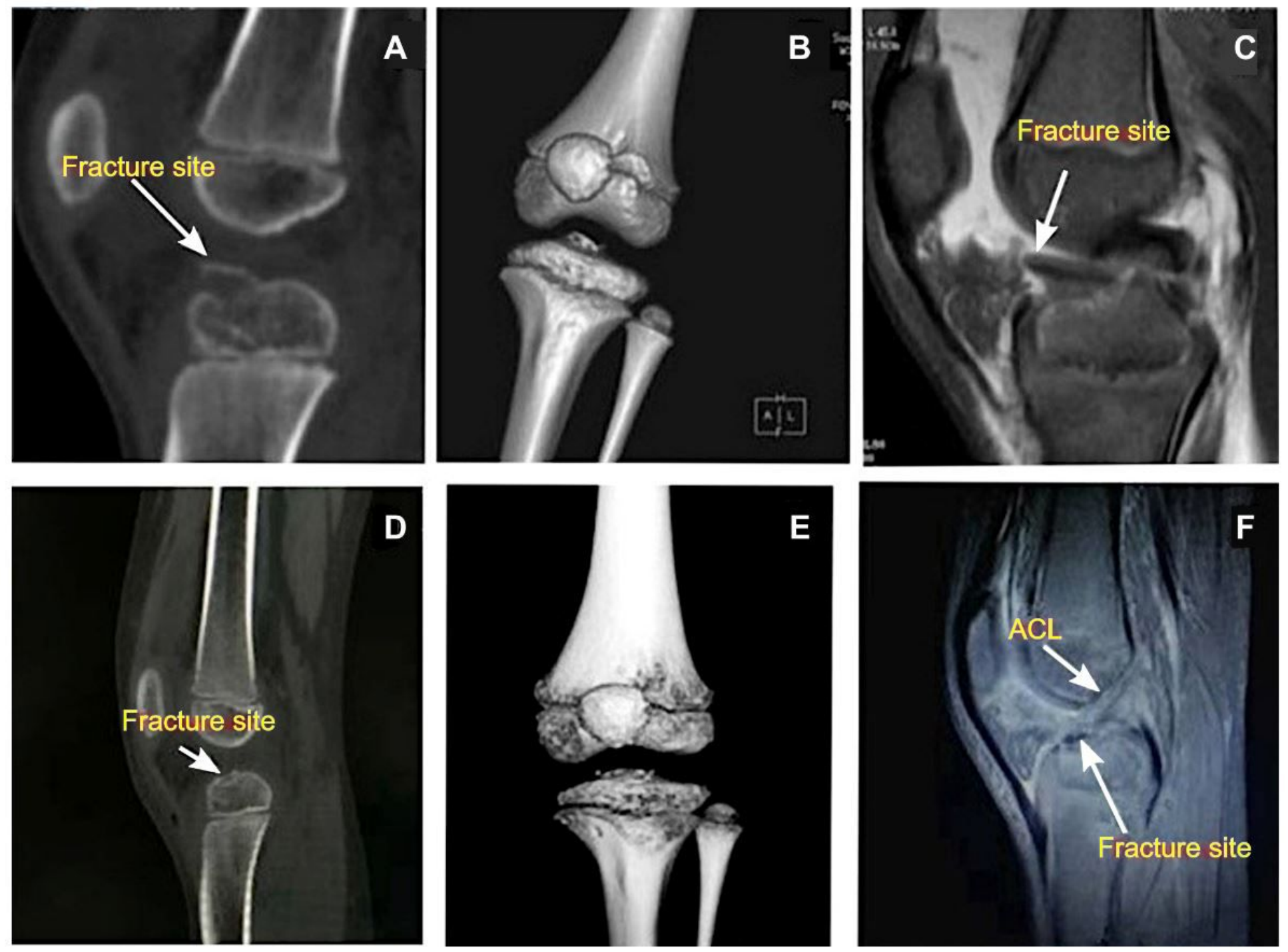

Figure 2

Pre- and post-operative imaging of anterior cruciate ligament (ACL) tibial avulsion fracture. A: Preoperative X-ray. ACL tibial fracture displacement (white arrow). B: Pre-operative 3D computed tomography (CT). ACL tibial fracture displacement. C: Pre-operative magnetic resonance imaging (MRI). ACL tibial fracture displacement, upturned (white arrow). D: Post-operative X-ray. Reduction of ACL tibial avulsion fracture (white arrow). E: Post-operative 3D CT. Reduction of ACL tibial avulsion fracture (white arrow). F: Post-operative MRI. Reduction of ACL tibial avulsion fracture, with good ACL continuity (white arrow). 